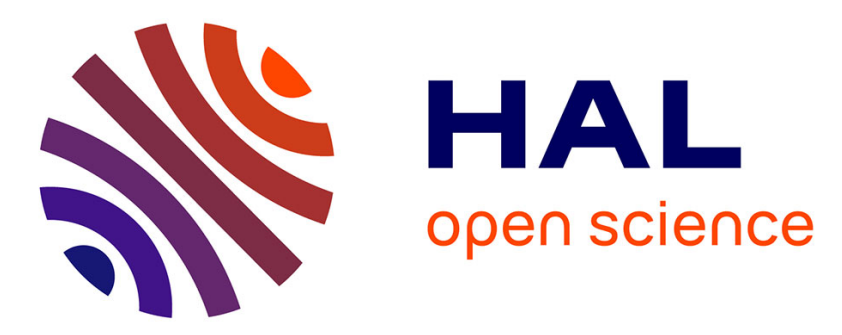

\title{
Dyes@BNNT Nanohybrids for Photostable Fluorescence Imaging
}

\author{
Charlotte Allard, Raffaela Nascimento, Frederic Fossard, Léonard Schué, \\ Emmanuel Flahaut, Annick Loiseau, Patrick Desjardins, Richard Martel, \\ Étienne Gaufrès
}

\section{To cite this version:}

Charlotte Allard, Raffaela Nascimento, Frederic Fossard, Léonard Schué, Emmanuel Flahaut, et al.. Dyes@BNNT Nanohybrids for Photostable Fluorescence Imaging. 2019. hal-02387311

\section{HAL Id: hal-02387311 https://hal.science/hal-02387311}

Preprint submitted on 29 Nov 2019

HAL is a multi-disciplinary open access archive for the deposit and dissemination of scientific research documents, whether they are published or not. The documents may come from teaching and research institutions in France or abroad, or from public or private research centers.
L'archive ouverte pluridisciplinaire HAL, est destinée au dépôt et à la diffusion de documents scientifiques de niveau recherche, publiés ou non, émanant des établissements d'enseignement et de recherche français ou étrangers, des laboratoires publics ou privés. 


\section{Dyes@BNNT Nanohybrids for Photostable Fluorescence Imaging}

Charlotte Allard, Raffaela Nascimento, Frederic Fossard, Leonard Schué, Emmanuel Flahaut, Annick Loiseau, Patrick Desjardins, Richard Martel, Etienne Gaufres

Submitted date: 18/04/2019 - Posted date: 19/04/2019

Licence: CC BY-NC-ND 4.0

Citation information: Allard, Charlotte; Nascimento, Raffaela; Fossard, Frederic; Schué, Leonard; Flahaut, Emmanuel; Loiseau, Annick; et al. (2019): Dyes@BNNT Nanohybrids for Photostable Fluorescence Imaging. ChemRxiv. Preprint.

Fluorescence is ubiquitous in life science and used in broad fields of research going from ecology to medicine. Among the most common fluorogenic compounds, dyes are being exploited in bioimaging for their outstanding optical properties across a broad range of wavelengths from the UV to the near-IR. However, dye molecules are often toxic to living organisms and photodegradable, giving limited time windows for in vivo monitoring. By encapsulating organic dyes inside a boron nitride nanotube (dyes@BNNT), we achieve a passivation of the dyes against photodegradation and chemical reaction. The dyes@BNNT nanohybrids contain aggregated and ordered dyes exhibiting strong photoluminescence with signal remaining stable and exempt of blinking over a time scale of more than 104 compared to free dyes. Our results also suggest reduced toxicity and exceptional chemical robustness even in harsh environments. The use of these 1D dyes@BNNT nanohybrids as fluorescence nanoprobes in bio-imaging is highlighted with in-vivo monitoring experiments on living Daphnia Pulex.

File list (2) 


\section{Dyes@BNNT Nanohybrids for Photostable Fluorescence Imaging}

Charlotte Allard ${ }^{1}$, Rafaella Nascimento ${ }^{2}$ Frédéric Fossard ${ }^{3}$, Léonard Schué ${ }^{2}$, Emmanuel Flahaut ${ }^{4}$, Annick Loiseau ${ }^{3}$, Patrick Desjardins ${ }^{1},{\text { Richard } \text { Martel }^{2 *} \text { and Etienne Gaufrès }}^{3,5 *}$

${ }^{1}$ Regroupement Québécois sur les Matériaux de Pointe (RQMP) and département de génie physique, Polytechnique Montréal, Montréal, Québec H3C 3A7, Canada

${ }^{2}$ Regroupement Québécois sur les Matériaux de Pointe (RQMP) and département de chimie, Université de Montréal, Montréal, Québec H3C 3J7, Canada

${ }^{3}$ Laboratoire d'Etude des Microstructures, ONERA-CNRS, UMR104, Université Paris-Saclay, BP 72, 92322 Châtillon Cedex, France

${ }^{4}$ CIRIMAT, Université de Toulouse, CNRS, INPT, UPS, UMR CNRS-UPS-INP N5085, Université Toulouse 3 Paul Sabatier, Bât. CIRIMAT, 118, route de Narbonne, 31062 Toulouse cedex 9, France

${ }^{5}$ Institut d'Optique \& CNRS, LP2N UMR 5298, F-33400 Talence, France

*Correspondence to: etienne.gaufres@u-bordeaux.fr and r.martel@,umontreal.ca 
Abstract.

Fluorescence is ubiquitous in life science and used in broad fields of research going from ecology to medicine. Among the most common fluorogenic compounds, dyes are being exploited in bioimaging for their outstanding optical properties across a broad range of wavelengths from the UV to the near-IR. However, dye molecules are often toxic to living organisms and photodegradable, giving limited time windows for in vivo monitoring. By encapsulating organic dyes inside a boron nitride nanotube (dyes@BNNT), we achieve a passivation of the dyes against photodegradation and chemical reaction. The dyes@BNNT nanohybrids contain aggregated and ordered dyes exhibiting strong photoluminescence with signal remaining stable and exempt of blinking over a time scale of more than $10^{4}$ compared to free dyes. Our results also suggest reduced toxicity and exceptional chemical robustness even in harsh environments. The use of these 1D dyes@BNNT nanohybrids as fluorescence nanoprobes in bio-imaging is highlighted with in-vivo monitoring experiments on living Daphnia Pulex.

Dye molecules produce intense absorption bands and fluorescence signals over a broad range of wavelengths from the near infrared (NIR) to the ultraviolet (UV). Because of these exceptional properties, they are widely used to color and emit light in various applications, going from dyeing fabrics to displays. Although they have become ubiquitous in our lives, organic dyes are inherently photodegradable and reactive in physiological conditions ${ }^{1}$. Known since the $19^{\text {th }}$ century ${ }^{2}$, the dye's instabilities stem in part from different photo-activated physical and chemical processes occurring during the lifetime of the dye's excited-state, which include dark state formation by 
intersystem crossing ${ }^{3,4}$, molecular conformation change ${ }^{5}$, and light-induced charging, resulting in temporary perturbations (blinking) due to random excursions between bright and dark states ${ }^{6-8}$. More significantly, oxidizing species (ROS) placed in contact with the dye induce irreversible photoluminescence (PL) extinction, known as photobleaching or fading ${ }^{9,10}$. The processes drastically reduce the time window to perform experiments and limit more particularly bioimaging applications such as in-vivo monitoring in various conditions. For example, the green fluorescent protein (GFP) provides a limited number of absorption/emission cycles in the range between $10^{4}$ to $10^{5}$ emitted photons before photo-bleaching. GFPs remain nevertheless very popular as fluorescence probes, but their use is limited to only a few minutes in typical imaging conditions $^{11,12}$.

Expedients to delay photobleaching have been developed and good examples include the addition of oxygen-blocking molecules, such as anoxia ${ }^{13}$, and a passivation of the dyes against ROS by a polymer, a porous material, or a supramolecular assembly ${ }^{14-16}$. These approaches have had, however, a limited success due to the size increase around the dyes and loss of brightness from the presence of quenchers in the passivation layers. As an example, inorganic calcium phosphate nanoparticles mixed with dyes (called FloDots) have been developed to prepare photostable fluorescence probes for imaging ${ }^{17}$, but the size constraints $(10-120 \mathrm{~nm})$ limit the diffusion properties of the probe.

Inspired by these passivation strategies and recent advances on the encapsulation of organic dyes inside single-walled carbon nanotubes ${ }^{18-21}$ (SWCNTs), we have explored the use of nanotubes as an alternative host to passivate dyes from the environment while keeping both small (nanometer) sizes and good optical properties (e.g. brightness and stability). Because of efficient energy transfer processes between dyes and SWCNT hosts $\left(\mathrm{E}_{\mathrm{g}}<1 \mathrm{eV}\right)$, it become apparent that 
the PL of the dyes is readily quenched in most, if not all, of these nanohybrids ${ }^{22,23}$. Hence, we turn our attention to the boron nitride nanotube $(\mathrm{BNNT})$, which has a band gap energy $\left(\mathrm{E}_{\mathrm{g}} \sim 5.5 \mathrm{eV}\right)$ that is large enough to be transparent within a wide range of wavelengths and quasi-independent from the chirality ${ }^{24,25}$. Herein, we report results on the preparation and on the chemical and photoluminescence properties of dyes encapsulated inside BNNTs (dyes@BNNTs). Our experiments on $\alpha$-sexithiophene (6T) and derivatives of 3,6-Bis[2,2']bithiophenyl-5-yl-2,5-di-noc-tylpyrrolo[3,4-c]pyrrole-1,4-dione (DPP) encapsulated inside small diameter BNNTs $\left(\mathrm{d}_{\text {inner }}<\right.$ $3 \mathrm{~nm}$ ) show a drastic improvement of the stability compared to free dyes against laser light exposure. We also observe effective passivation of the dyes against the environment, even under harsh chemical conditions. Using various imaging demonstrations with living organisms (Daphnia Pulex), we report on a reduced toxicity of the dye nanohybrid compared to free dyes and demonstrate their exceptional photostability as a nanoprobe for multi-modal bio-imaging in a wide range of wavelengths.

\section{Results and discussions}

Figure 1 schematizes the main synthesis steps of the dye nanohybrids along with the photoluminescence response obtained before and after the dye encapsulation. The synthesis begins by cutting raw BNNTs (BNNT LLC supplier) using mechanical grinding and ultrasound treatments. The resulting material is then purified in nitric acid and annealed at high temperature in air $^{26}$ to suppress background PL from $\mathrm{BN}$ and $\mathrm{B}_{\mathrm{x}} \mathrm{O}_{\mathrm{y}}$ impurities ${ }^{27}$ (Supplementary Fig. 1). The last step is a liquid phase encapsulation of a selected dye molecule, e.g. DPP2 (Fig. 1a), in thus processed BNNTs, followed by a thorough rinsing in DMF/Toluene and finally with a piranha solution at room temperature to remove excess of dye molecules. The resulting solid can be dispersed in a solvent and yields a highly colored solution, such as shown in Fig. 1a and c. 
The first signature of encapsulation is a clear color change compared to that of free dyes. In Fig. 1c, the coloration of the free $6 \mathrm{~T}$ in DMF (P1) is compared to that of a mixture of $6 \mathrm{~T}$ and BNNTs after an encapsulation step in toluene for 24 hours at $25^{\circ} \mathrm{C}(\mathrm{P} 2)$ and $115^{\circ} \mathrm{C}(\mathrm{P} 3)$. No change is observed between P1 and P2, whereas a clear color change from yellow to red is observed for P3. As detailed below, the color shift indicates that the dyes have filled the BNNTs and the encapsulation is driven by temperature. Similar spectral changes of the dye resonance are also observed with other rod-like molecules. For example, the derivatives of 3,6-Bis[2,2']bithiophenyl5-yl-2,5-di-n-oc-tylpyrrolo[3,4-c]pyrrole-1,4-dione (DPP2 and DPP3) are encapsulated using the same protocol but at $80^{\circ} \mathrm{C}$ (Fig. 1c and Supplementary Section 2.2). The behavior observed here with BNNTs is fully consistent with previous results of dye encapsulation in SWCNTs ${ }^{22}$. For both types of nanotubes, the process is endothermic, a behavior ascribed to a heat unbalance from intermolecular interactions between dyes (encapsulated) molecules and between dyes (free) and solvent molecules.

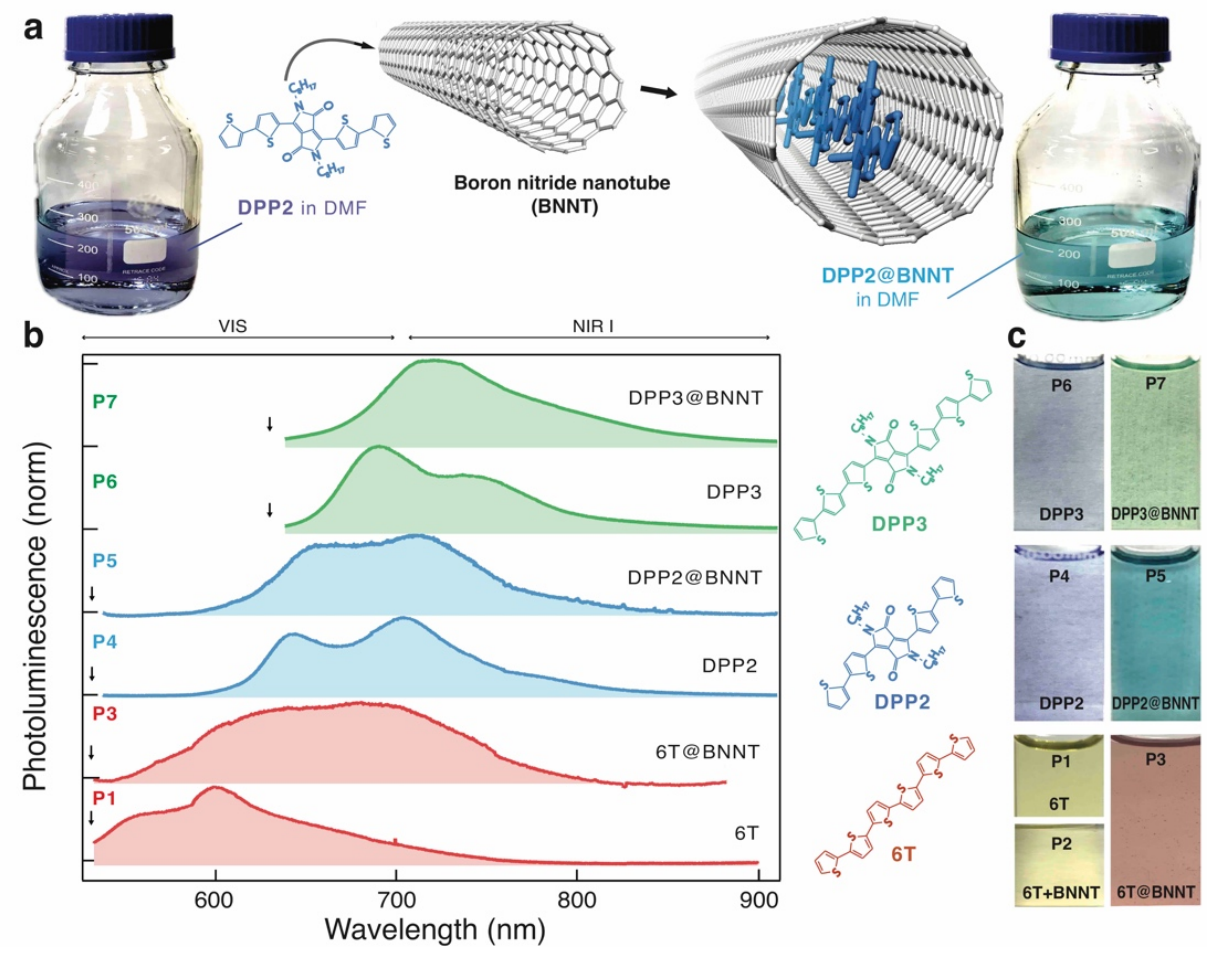




\section{Figure 1: Photoluminescence properties of 6T@BNNTs, DPP2@BNNTs and DPP3@BNNTs.}

a, Schematic view of the encapsulation of DPP2 in a double-wall BNNT and the resulting color change before and after encapsulation in DMF. b, Photoluminescence spectra at room temperature of the solutions P1, P3-P5 at an excitation wavelength $\left(\lambda_{\mathrm{ex}}\right)$ of $532 \mathrm{~nm}$ and P6-P7 at $\lambda_{\mathrm{ex}}=633 \mathrm{~nm}$. c, Schematics of 6T and DPP based dyes molecules (left) and pictures of the dye solutions in DMF before and after a liquid phase encapsulation (in toluene) at different temperatures: $25^{\circ} \mathrm{C}(\mathrm{P} 2)$ and $115^{\circ} \mathrm{C}(\mathrm{P} 3)$ for $6 \mathrm{~T}$ and $80^{\circ} \mathrm{C}$ for DPPs (P5 and P7). P1, P4 and P6 are solutions of free dyes (no BNNT) in DMF.

Both a shift in color and an endothermic process of encapsulation imply realignment and aggregation of the dyes within the inner space of the nanotubes. In Figures 1b, we explore more quantitatively this transformation using different dye nanohybrids (P3, P5 and P7) in DMF compared to the solutions of the same dyes (free) in DMF (P1, P4, P6). For example, a solution of free 6Ts (P1) displays a photoluminescence line shape characterized by a strong $0-0$ vibronic band. In contrast, the6T@BNNT solution (P3) displays a PL spectrum that is red-shifted and broadened to a point that vibronic bands become undistinguishable. Similar behavior is observed with DPP2 and DPP3 and DPP@BNNT solutions. A strong 0-0 vibronic band is related to energetic disorders between free molecules, which is typical of individualized dye molecules in polymers, such as polymethylmethacrylate ${ }^{28,29}$ (PMMA). For all of the dye nanohybrids tested so far, we observed no clear vibronic structure and a slight translucence of the solutions ascribed to molecular ordering (dye aggregation) and optical diffusion induced by the BNNT hosts. 
The passivation of the encapsulated dyes with the crystalline dielectric sheath of the BNNT walls is tested next using stability measurements against photo-bleaching and chemical reaction. To do so, we compared in Fig. 2 the PL properties of free dyes with that of individual or small bundles of $6 \mathrm{~T} @ \mathrm{BNNTs}$ deposited on an inert $\mathrm{Si} / \mathrm{SiO}_{2}$ substrate in ambient conditions. Note that an oxide thickness of $\sim 300 \mathrm{~nm}$ is chosen to avoid destructive optical interference with the substrate. To gain statistics, a global hyperspectral imager (RIMA ${ }^{\mathrm{TM}}$, Photon etc.) was used to photo-excite uniformly a large area of the sample $\left(200 \times 200 \mu \mathrm{m}^{2}\right)$ with continuous laser light ${ }^{30}$ at $\lambda_{\mathrm{ex}}=532 \mathrm{~nm}$. This configuration has given us a large statistical ensemble of measurements on individualized 6T@BNNT bundles as a function of exposure time and fluence conditions. As an example, Figure 2a shows a zoomed view of the PL image of a $5 \mu \mathrm{m}$ long bundle at a laser fluence typical for bioimaging $\left(1.2 \mu \mathrm{W} . \mu \mathrm{m}^{-2}\right)$. In Figure $2 \mathrm{~b}$, the PL signal of the bundle, as a function of exposure time, shows no noticeable intensity loss, even after exposure for more than 8 hours in air. This interesting result indicates a clear resistance against photobleaching, which is also demonstrated with the DPP nanohybrids in Fig. 2b. The result contrasts with the fast fading PL signal (in red) of free dyes (not encapsulated) dispersed on the substrate. As expected and typical of photobleaching, the half lifetime of the integrated emission $\left(\tau_{1 / 2}\right)$ of free dyes is only few minutes, whereas individual dyes@BNNT bundles produce unaltered PL after more than 30 hours, which is only limited here by our setup to a factor of $1 \times 10^{4}$. Furthermore, PL imaging taken at the level of a single dye nanohybrid with a frame rate of $0.5 \mathrm{sec}$ indicates no blinking under a fluence of $1.2 \mu \mathrm{W} . \mu \mathrm{m}^{-2}$ at $\lambda_{\mathrm{ex}}=532 \mathrm{~nm}$ (Fig 2c). These photostability results are impressive considering that this is a lower bound estimate limited only by our setup.

The chemical stability of the dye nanohybrids is tested using individualized 6T@BNNT bundles deposited on $\mathrm{Si} / \mathrm{SiO}_{2}$ by exposure to an oxygen plasma of $100 \mathrm{~W}$ for 10 minutes. No significant 
change in the PL spectral shape and intensity is detected after treatment, whereas free 6T molecules on $\mathrm{Si} / \mathrm{SiO}_{2}$ have completely vanished (Fig. $2 \mathrm{~d}$ ). The enhanced stability of the encapsulated dyes has also been observed upon thermal annealing up to $350^{\circ} \mathrm{C}$ in air and using piranha solutions (not shown). These results demonstrate that the 6T@BNNT is far more stable than free 6T dyes. The chemical and physical stability experiments have been repeated on a number of dye nanohybrids of different lengths deposited on substrates or dispersed in a solution (See e.g. Supplementary Figs. 5 and 6). All of the results share the same conclusion, which underlines the tremendous gain in stability due to dye confinement inside the 1D cavity of BNNTs.
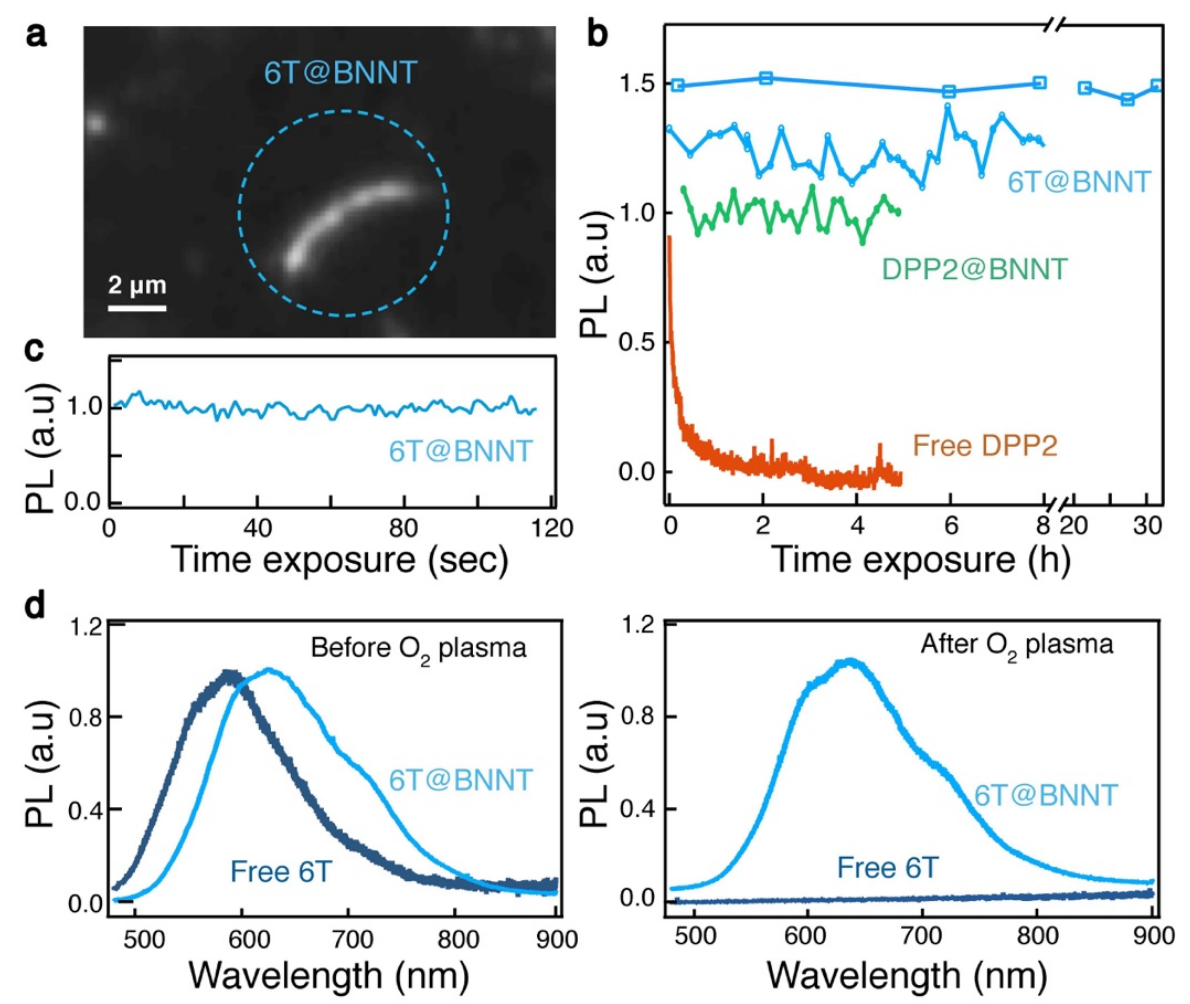

Figure 2: Photostability and chemical resistance of 6T@BNNTs. a, Integrated luminescence imaging of a typical microbundle of a $6 \mathrm{~T} @ \mathrm{BNNT}$ deposited on a $\mathrm{Si} / \mathrm{SiO}_{2}$ substrate $\left(\lambda_{\mathrm{ex}}=532 \mathrm{~nm}\right)$. The time acquisition is $0.2 \mathrm{sec}$ and the laser fluence is $1.2 \mu \mathrm{W} . \mu \mathrm{m}^{-2}$. b, Time evolution of the 
integrated PL of isolated bundles of 6T@BNNTs and DPP2@BNNTs on a $\mathrm{Si} / \mathrm{SiO}_{2}$ surface, compared to free DPP2 photoexcited under a fluence of $1.2 \mu \mathrm{W} \cdot \mu \mathrm{m}^{-2}$ at $\lambda_{\mathrm{ex}}=532 \mathrm{~nm}$ (long pass filter at $533 \mathrm{~nm})$. c, PL intensity recorded at time intervals of $0.5 \mathrm{sec}$. d, PL spectra $\left(\lambda_{\mathrm{ex}}=473 \mathrm{~nm}\right)$ of free 6T and6T@BNNT deposited on a substrate recorded before (left) and after (right) a 100 W treatment to oxygen plasma for 10 minutes.

The changes in optical and chemical properties of dye molecules, as reported in Figs 1 and 2, stem from specific structural rearrangements of the dyes driven by the BNNT template. These morphological rearrangements of the dyes form aggregates that can be directly observed in High Resolution Transmission Electron Microscopy (HRTEM). Examples in Fig. 3 show images of 6T molecules encapsulated inside double- and multi-walled BNNTs deposited from solutions onto a Molybdenum- $\mathrm{SiO}_{2}\left(\mathrm{Mo} / \mathrm{SiO}_{2}\right)$ TEM grid. More examples of HRTEM are also shown in Supplementary, Fig. S8. A progressive ordering of the 6T dyes from disordered assemblies to wellaligned individual molecules is observed for inner diameters $\left(\mathrm{d}_{\text {inner }}\right)$ going from $7 \mathrm{~nm}$ down to 0.9 nm, respectively (Fig. 3f). A key result for this study, which is shown schematically in Figure 3A, is the presence of single and double aggregates of 6T molecules in BNNTs having the smallest

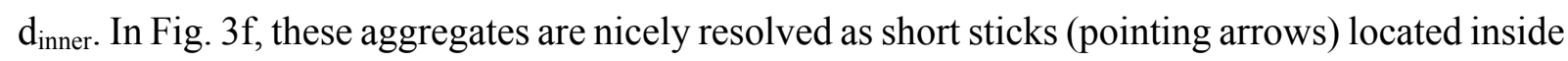
BNNTs of $d_{\text {inner }}=0.9$ and $1.55 \mathrm{~nm}$ for single and double aggregates, respectively. It is clear from the images that high confinement (i.e. low $\mathrm{d}_{\text {inner }}$ ) induces molecular ordering and alignment along the nanotube. A cavity of $d_{\text {inner }}=4 \mathrm{~nm}$ is, for instance, much larger (about twice) than the length of the molecules and provides higher degrees of freedom to accommodate various assemblies, whereas $\mathrm{d}_{\text {inner }}=0.9 \mathrm{~nm}$ (the smallest diameter of our sample) provides just enough space to fit one molecule in a head-to-tail type of stacking. 
To determine that the filling material is composed of 6T molecules, complementary experiments were performed using Scanning Transmission Electron Microscopy and Energy Electron Loss Spectroscopy (STEM-EELS) acquired at $80 \mathrm{kV}$ to gain information on the chemical composition (core-loss) and near-band edge response (low-loss) of the filling materials with a spatial resolution of around $2.5 \mathrm{~nm}$ (Supplementary Fig. 7). The HRTEM image in Fig. 3b provides distinct responses in the empty and filled regions (open circles 1 and 2, respectively), which are presented in Figs 3c-e. While the boron K-edge from the shell structure of BNNT at $192 \mathrm{eV}$ is, as expected, measured in both regions 1 and 2, a new contribution is detected only in region 1 at the energy corresponding to the L2,3-edge of the sulfur atoms of $6 \mathrm{~T}$ molecules. Local energy loss measurements in regions 1 and 2 (Fig. 3e) show the expected transitions at around $3 \mathrm{eV}$ (region 1) for the HOMO-LUMO gap of 6T molecules and at $5.5 \mathrm{eV}$ (region 2) for the BNNT band gap. Consistent to other reports on similar oligothiophene encapsulation inside SWCNTs ${ }^{19,20,31,32}$, the results confirm the encapsulation of the 6Ts inside BNNTs. 

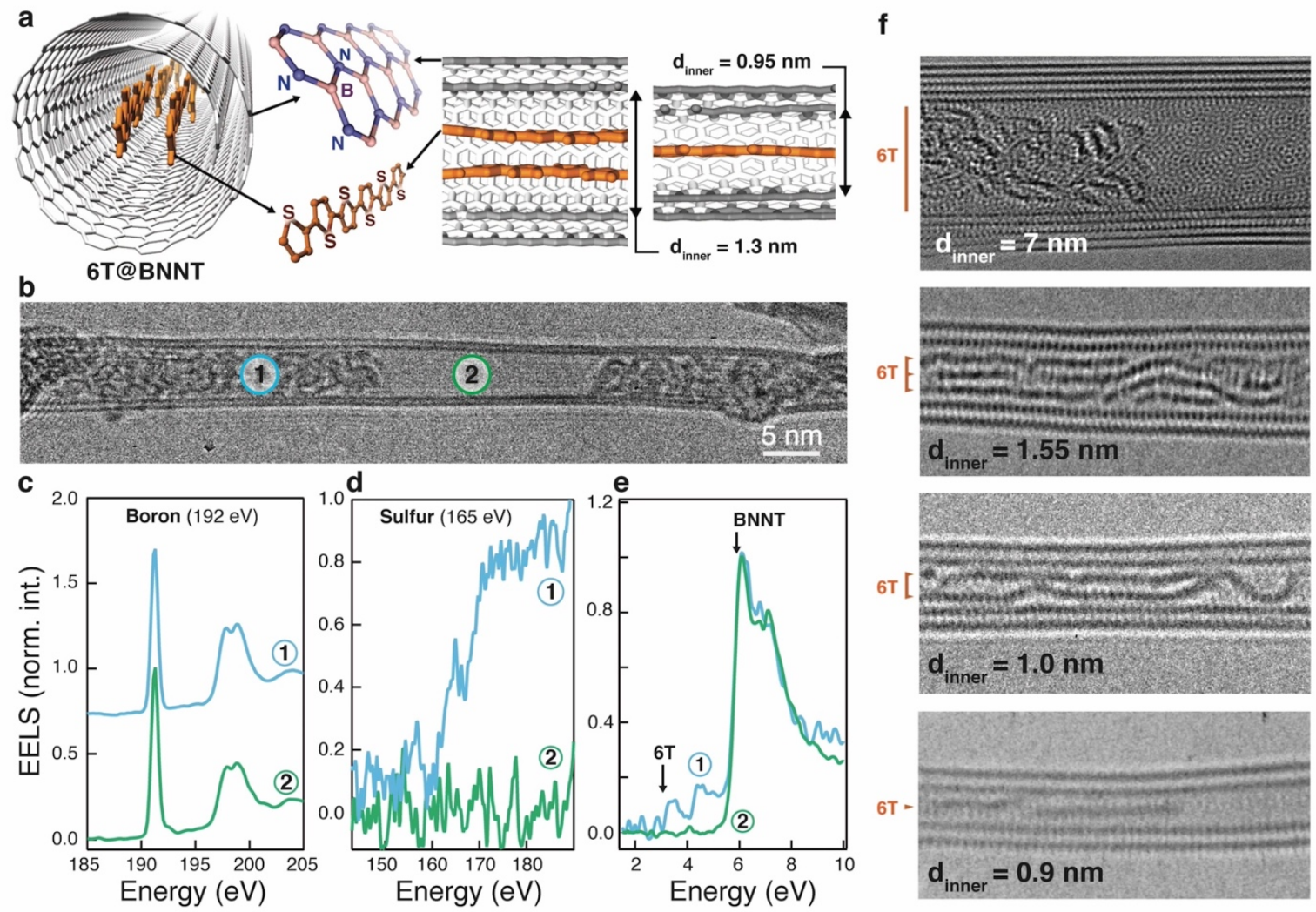

Figure 3: Structural properties of $\alpha$-sexithiophene (6T) encapsulated inside a double-walled

boron nitride nanotubes (6T@BNNTs). a, Schematic view of the structure of 6T molecules encapsulated in double-wall BNNTs.b, HRTEM image of a partially filled 6T@BNNT suspended on a Molybdenum-SiO $\mathrm{S}_{2}$ TEM grid. $\mathbf{c}$ and $\mathbf{d}$, Chemical analysis of selected areas (1) and (2) in (b) using core-loss STEM-EELS at the boron K-edge (192 eV) and sulfur L2,3-edge (165 eV). e, Measurements of the energy levels in the same two areas by STEM-EELS in the 1-10 eV energy range. f, HRTEM images of 6T molecules inside BNNTs of different diameters. The HRTEM and STEM-EELS data are recorded at $80 \mathrm{kV}$. 
The assembly of dyes inside BNNTs into aligned and structured aggregates is consistent with the assembly behavior observed on elongated dyes encapsulated inside SWCNTs ${ }^{19-22,31,33}$. The similarities between these systems are striking, as both provide higher stability against photodegradation and exposure to ROSs. As seen in the HRTEM images of Fig. 3f, the head-totail stacking of the encapsulated dyes in small diameter BNNTs corresponds to the dye morphology of the emblematic J-aggregate state, a highly emissive state. On the contrary and not seen in our samples, a face-to-face stacking is characteristic of a low emissive state, the $\mathrm{H}$ aggregate. These aggregation states greatly influence the photo-physical properties of the dyes though interaction due to e.g. interaction and energy shifts associated with Davidov splitting ${ }^{34,35}$. We suspect that the overall red shift observed in Fig. 1 originates from J-like aggregations of the confined dyes and that the broadening of the bands is due to the large distribution of $\mathrm{d}_{\text {inner }}$ in our samples, as evidenced by HRTEM in Fig. 3. Hence, the broad PL envelopes observed with different dyes nanohybrids are most likely due to the size distribution of $\mathrm{d}_{\text {inner }}$, giving a convolution of an ensemble of individual emitters having each a specific spectrum and aggregation state. Further PL work on dye nanohybrids with diameter sorted BNNTs will be required to analyze the vibronic fingerprints of each emitter as a function of confinement and ordering. Nevertheless, a sorting of the nanotubes by diameter appears a valid approach to control the aggregation state and emissive properties of the dyes.

The impressive robustness and fading free properties of the Dyes@BNNT are appealing for bioimaging applications. Figure 4 presents an example of the use of dye nanohybrids to make optical nanoprobes for imaging living Daphina Pulex organisms in water - see bright-field image in Figure 4a\#1. Daphnia (also called water fleas) are common planktonic crustaceans, which find use as chemical sensors in ecotoxicology studies, thanks to their high sensitivity to water quality ${ }^{36}$. To 
acquire the bio-images (Fig. 4a\#2-5), we first solubilized the prepared dye nanohybrids (dye $@ B N N T)$ in a water based living medium called LLAMES $^{37}$, using a poly (ethylene) glycol derivative (mPEG-DSPE) (Supplementary Section 2.9). We then performed a first incubation test by placing a Daphnia in different FLAMES containing either free 6T, only PEG-BNNTs, or PEGdyes@BNNTs in different concentrations (see Supplementary Table S1). After an incubation of only few minutes, all daphniids exposed to free $6 \mathrm{~T}$ were found dead. This is consistent with the known toxicity of such dyes to living organisms, which was evidenced in the $20^{\text {th }}$ century by many studies on drugs or contrast agents ${ }^{38}$. On the contrary, all of the Daphnia assays incubated with both PEG-BNNT and PEG-dyes@BNNT remain alive and active after incubation times ranging from a few minutes to 36 hours at concentrations between 10 and $50 \mu \mathrm{g} \cdot \mathrm{ml}^{-1}$ (see video in Supplementary Fig. 4). This underlines the multiple roles of the BNNT host: i) Stabilize the PL signal from the nanoprobe, ii) act as a protective barrier against ROS, and iii) reduce toxicity to the living organisms. Living daphniids were then imaged under a fluorescence microscope equipped with UBG filter and a 10X objective. Daphnia Pulex incubated with PEG-BNNTs (i.e. no dye, Fig. 4a\#2 down) presents similar signal than that observed from non-incubated Daphnia (Fig. $4 \mathrm{a} \# 2$ top). This natural blue-green signal originates from the auto-fluorescence of the tissues in FLAMES through the UBG filter, which disappears after death or after few minutes only of photobleaching. In contrast, Daphniids incubated with PEG-Dyes@BNNTs (Fig.4a\#3 - 5) exhibit a distinct digestive tube emitting bright signals assigned to the spectral lineshape of each PEGDyes@BNNTs nanohybrids. 

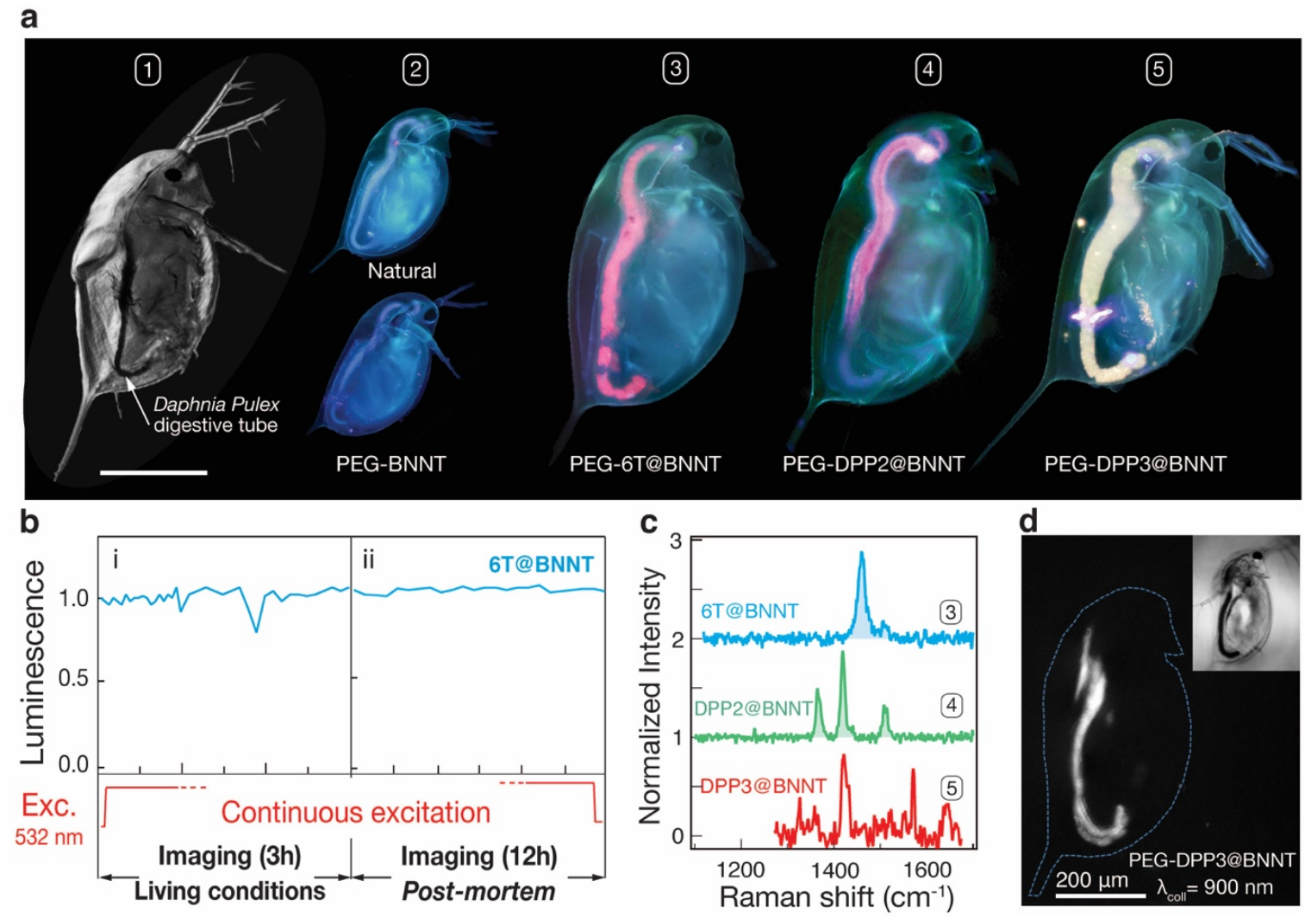

Figure 4: Dyes@BNNTs as hypermodal nanoprobes illustrated for Vis-NIR bio-imaging of

Daphnia Pulex. a, Z-reconstructed confocal optical image of a Daphnia immobilized in Leica medium (\#1). PL images recorded through a fluorescence microscope mounted with a UBG filter and 10X objective lens of living Daphnia (top \#2) and incubated in a solution of unfilled BNNTs (bottom \#2),6T@BNNTs (\#3), DPP2@BNNTs (\#4) and DPP3@BNNTs (\#5).b, Luminescence intensity time-lapses of dyes@BNNTs, (i) in living conditions, (ii) in post-mortem conditions. c, Raman spectra at $\lambda_{\text {ex }}=532 \mathrm{~nm}$ taken in the region of the digestive tube of Daphnia for \#3 and at $\lambda_{\text {ex }}=633 \mathrm{~nm}$ for \#4 and \#5. d, PL image of a living Daphnia collected at a wavelength of $900 \mathrm{~nm}$ using an excitation at $\lambda_{\mathrm{ex}}=532 \mathrm{~nm}$. Inset: Optical image of the Daphnia. The scale bar is $200 \mu \mathrm{m}$ for images \#1, \#3-\#5 and $400 \mu \mathrm{m}$ for \#2. 
In a second set of experiments, we investigated the photo-stability of the nanoprobes during invivo PL imaging of Daphnia Pulex under continuous light excitation. Daphniids were maintained alive during the first 3 hours of the time-lapse by adding more FLAMES to maintain temperature and compensate water evaporation under the microscope. The PL intensity, recorded every 3 minutes at 6 different points of the digestive tube (Fig. 4a), remains flat with time, which indicates that bleaching is suppressed in such continuous photoexcitation despite the fact that the nanoprobes are being in close contact with the ROS present in the water-based medium of the digestive tube of Daphniids. The brief and temporary drops of signal at $1 \mathrm{~h}$ and $3 \mathrm{~h}$ are attributed to sudden movements of the Daphnia in the exiguous swimming pool under the microscope objective (Fig. 4b). Even after death, which is typically caused by a drying of the FLAMES medium, the signal of the dye nanohybrids remain stable for an additional 12 hours of continuous excitation/imaging without any noticeable loss of signal (Fig. 4b). Interestingly, the Raman spectra in Fig. 4c, recorded at the level of the digestive tube of Daphnia $\# 4$ and $\# 5$, show that the specific vibrational fingerprint of the encapsulated 6T (or DPP) in BNNTs remain intact. This signal can be readily extracted and identified from the luminescence signal, thanks to the resonance enhancement of the dyes at $\lambda_{\text {ex }}=$ $532 \mathrm{~nm}$. This last experiment demonstrates that the encapsulated dyes are not altered in the digestive tube of the living Daphnia and that they are highly photo-stable, giving further statistics of the effective passivation by the BNNT hosts.

The capability to probe in-situ both the photoluminescence and Raman scattering of different nanoprobes over a timescale of many hours is an interesting aspect with dye nanohybrids for use as multimodal probes for bio-imaging. Similar to the work on carbon nanotubes based NIR nanoprobes $^{39-42}$, which emit at wavelengths beyond $900 \mathrm{~nm}$, we performed experiments using a NIR DPP3@BNNT incubated in Daphnia. By simply shifting dyes, we were able to extend the PL 
emission (Fig. 4d) to the NIR I optical bio-window ${ }^{43}$. We trust that this simple approach can be expanded further to other dyes (even toxic) offering broad NIR emission wavelengths. This will provide probes for deeper analysis in the near-infrared optical windows ${ }^{44}$. In addition, these dyes nanohybrids, because of their 1D architecture, have similar diffusion properties than SWCNTs, which are advantageous for studies in biological media ${ }^{45,46}$.

Previous works on the functionalization of multi-walled BNNTs have shown that BNNTs are appropriate templates to develop active nanostructures, such as drug delivery and other bioinvestigations ${ }^{47-49}$. Here, we have highlighted the potential use of dyes@BNNT nanohybrids to make active nanoprobes with high brightness and exceptional stability and tunability. Building on the constantly evolving knowledge on BNNT materials, it is expected that the dye@BNNT nanohybrids will greatly impact bio-imaging by extending the acquisition time and optical windows into the NIR I and II to go deeper into the body of living organisms. Tailoring optical signatures with enhanced stability through the 1D aggregation of dyes inside BNNT will represent a paradigm shift in developing for instance new in-vivo and post mortem biological investigations that have not yet been possible due to the limitations of conventional fluorescence probes.

\section{Methods}

The boron nitride nanotubes (BNNT) used in this work are provided by $\mathrm{BNNT}^{\mathrm{TM}}$. See Supplementary Section 2.1 and 2.2 for details on cleaning and encapsulation of BNNTs. Briefly, the BNNT powder was sonicated in dimethylformamide (DMF) until complete dispersion, and filtrated on a PTFE filter $(0.2 \mu \mathrm{m}$ pore size $)$. The obtained film was annealed at $800^{\circ} \mathrm{C}$ for $2 \mathrm{~h}$ under atmospheric conditions. About $5 \mathrm{mg}$ of dyes and $20 \mathrm{mg}$ of purified BNNTs were dispersed sequentially in $20 \mathrm{~mL}$ or $300 \mathrm{~mL}$ of toluene, depending on the dye used, by sonication. The solution 
was then refluxed at $80^{\circ} \mathrm{C}$ or $115^{\circ} \mathrm{C}$, again depending of the dyes used, to activate the encapsulation. The solution was finally washed in toluene and DMF at least 10 times to remove the free dyes molecules, until the filtrate became colorless.

For experiments on individualized Dyes@BNNTs on surface, the Dyes@BNNTs were suspended in $N, N$-dimethylformamide (DMF) and diluted as needed, typically at a concentration of $\sim 0.1$ $\mathrm{mg} / \mathrm{mL}$. This solution was spin-coated at $3000 \mathrm{rpm}$ on a cleaned $\mathrm{Si} / \mathrm{SiO}_{2}$ substrate with aminopropyltriethoxysilane (APTES) functionalization.

For experiment with Daphnia, an incubation solution was prepared by dispersing Dyes@BNNT in FLAMES culture medium at a concentration ranging from 5 to $100 \mu \mathrm{g} / \mathrm{ml}$. Adult individuals of Daphnia Pulex (clones DISP 1312) were kept at $22^{\circ} \mathrm{C}$ in FLAMES with algae for 4 days before the experiment, allowing for a new reproducing cycle. Then, the specimens were transferred to a FLAMES medium algae-free and remained there for 1 day at minimum to clean the digestive system and limit any PL from algae during the images/time lapse acquisition. Daphniids are then placed in the incubation solution for the required time. More details on the experiments with Daphnia and characterizations of the samples are provided in Supplementary Section 2.9 and 3. The AFM images were produced using a Dimension 3100 microscope. The Raman/PL measurements were taken from different set-up depending on the experiments and these instruments and conditions are summarized in Table S2. In summary, luminescence images and photobleaching experiments were performed at $\lambda_{\mathrm{ex}}=532 \mathrm{~nm}$ using the Raman/PL mapping system $\mathrm{RIMA}^{\mathrm{TM}}$ (Photon Etc) using a 100X objective and a laser power ranging from $0.5 \mu \mathrm{W} . \mu \mathrm{m}^{-2}$ to 80 $\mu \mathrm{W} \cdot \mu \mathrm{m}^{-2}$. Other fluorescence images were acquired with an Olympus confocal microscope equipped with a U-RFL-T mercury light source, a BX-UCB controller and a DP71 digital camera. The images were acquired with a 10X objective and UBG, TRITC and TXRED filters. The 
acquiring conditions were: Exposure time: 1/20 sec and ISO 200 sensitivity. Z-stack of 20 images to probe the volume of the Daphnia was acquired by confocal imaging and stacked using the Autoblend function in Phostoshop.

\section{Acknowledgments}

This work received financial support from the Natural Sciences and Engineering Research Council of Canada (NSERC), the Canada Research Chairs (CRC) programs. CA, RN, PD and RM acknowledge support from the consortium industriel de recherche et d'innovation en technology médicales du Québec (MEDTEQ) in collaboration with our partners Photon Etc. and La fondation du cancer du sein du Québec. No 706476 and Graphene Flagship Core 1 (No. 604391). EG acknowledges funding from the Marie-Skłodowska-Curie-IF 706476-BrightPhoton, the GDRi Graphene and Co and the GDRi multifunctional nano for travel support. The authors warmly acknowledge G. WANG for support on the Cs-corrected TEM of MPQ - Paris Diderot University and the METSA support for the access to the Cs-corrected TEM of MPQ. The authors would like to thank Cristescu's team (McGill University) for providing Daphniids, culture medium (FLAMES), algae and technical assistance, and help from L. Gaboury, J. Hinsinger and the Platform of Histology at Institut de Recherche en Immunologie et en Cancerologie (IRIC). The authors acknowledge BNNT LLC for providing raw BNNTs.

\section{Authors Contributions}

C.A, R.N, F.F, L.S, A.L, R.M, E.G performed the experiments and analysed the results, C.A, R.N, R.M, E.G designed the experiments, C.A, R.N, E.F, E.G prepared the samples, P.D, R.M and E.G 
supervised the work and discussed the results. All authors contributed to the scientific discussions, manuscript preparation and final version.

\section{Competing Interest Statement}

The authors declare no competing financial interests.

\section{References}

1. Marx, V. Probes: paths to photostability. Nat. Methods, 12, 3 (2015)

2. Dufton, A. The fading of colours. J. Soc. Dyers Colourist 10, 90-95 (1894).

3. Widengren, J. Mets, Ü. \& Rigler R. Fluorescence Correlation Spectroscopy of triplet states in solution: A theoretical and experimental study. J. Phys. Chem 99, 13368-13379 (1995)

4. Kasha, M. Characterization of electronic transitions in complex molecules. Discussion of the Faraday Society 9, 14-19 (1950)

5. Di Paolo, R. E. Scaffardi, L. B. Duchowicz, R. \& Bilmes, G. M. Photoisomerization Dynamics and spectroscopy of the Polymethine Dyes DTCI. J. Phys. Chem. 99, 13796$13799(1995)$

6. Moerner, W. E. \& Orrit, M. Illuminating single molecules in condensed matter. Science 283, $1670-1676(1999)$

7. Efros, A. L. \& Rosen, M. Random Telegraph Signal in the Photoluminescence Intensity of a Single Quantum Dot. Phys. Rev. Lett 78, 1110-1113 (1997) 
8. Galland, C. et al. Two types of luminescence blinking revealed by spectroelectrochemistry of single quantum dots. Nature 479, 203-207 (2011)

9. Widengren, J. \& Rigler, R. Mechanism of photobleaching investigated by fluorescence correlation spectroscopy. Bioimaging 4, 149-157 (1996)

10. Stennett, E. M. S. Ciuba, M. A. \& Levitus, M. Photophysical processes in single molecule organic fluorescent probes. Chem. Soc. Rev. 43, 1057-1075 (2014)

11. Diaspro, A. Chirico, G. Usai, C. Ramoino, P. \& Dobrucki, J. Photobleaching. Handbook of Biological Confocal Microscopy, J. B. Pawler, Springer New York (2006)

12. White, J. \& Stelzer, E. Photobleaching GFP reveals protein dynamics inside live cells. Cell Biology 9, $61-65$ (1999)

13. Bernas, T. Zarebski, M. Cook, R. R. \& Dobrucki, J. W. Minimizing photobleaching during confocal microscopy of fluorescent probes bound to chromatin: role of anoxia and photon flux. Journal of Microscopy 215, 281- 296 (2004)

14. D. Thomsson, et al. Cyclodextrin Insulation Prevents Static Quenching of Conjugated Polymer Fluorescence at the Single Molecule Level. Small 9, 15, 2619-2627 (2013)

15. Lee, K. J. Oh, J. H. Kim, Y. \& Jang, J. Fabrication of Photoluminescent Dyes/Poly (acrylonitrile) Coaxial Nanotubes Using Vapor Deposition Polymerization. Chem. Mater. 18, $5002-5008(2006)$

16. Frampton, M. J. \& Anderson, H. L. Insulated Molecular Wires. Angew. Chem. Int. Ed. 46, $1028-1064(2007)$

17. Yao, G. et al. FloDots: luminescent nanoparticles. Anal Bioanal Chem 385, 518-524 (2006) 
18. Yanagi, K. Miyata, Y. \& Kataura, H. Highly Stabilized $\beta$-Carotene in Carbon Nanotubes. Adv. Mater. 18, 437-441 (2006)

19. Loi, M. A. et al. Encapsulated of conjugated oligomers in single-walled carbon nanotubes towards nanohybrids for photonic devices. Adv. Mater. 22, 1635-1639 (2010)

20. Gaufrès, E. et al. Giant raman scattering from J-aggregated dyes inside carbon nanotubes for multispectral imaging. Nature Photonics 8, 72-78 (2014)

21. Cambré, S. et al. Asymetric dyes align inside carbon nanotubes to yield a large nonlinear optical response. Nat. Nanotech. 10, 248-252 (2015)

22. Gaufrès, E. et al. Aggregation control of $\alpha$-Sexithiophene via isothermal encapsulation inside single-walled carbon nanotubes. ACS Nano 10, 10220-10226 (2016)

23. van Bezouw, S. et al. Diameter-dependant optical absorption and excitation energy transfer from encapsulated dyes molecules toward single-walled carbon nanotubes. ACS Nano 12, 7, 6881-6894 (2018)

24. Blase, X. Rubio, A. Louie, S. G. \& Cohen, M.L. Stability and band gap constancy of boron nitride nanotubes. Europhys. Lett. 28, 5, 335-340 (1994)

25. Arenal, R. et al. Electron energy loss spectroscopy measurement of the optical gaps on individual boron nitride single-walled and multiwalled nanotubes. Phys. Rev. Lett. 95, $127601(2005)$

26. Chen, H. Chen, Y. Yu, J. \& Williams, J. S. Purification of boron nitride nanotubes. Chem. Phys. Lett. 425, 315-319 (2006) 
27. Chejanovsky, N. et al. Quantum ligth in curved low dimensional hexagonal boron nitride systems. Sci. Rep. 7, 14758 (2017)

28. Spano, F. C. Clark, J. Silva, C. \& Friend, R. H. Determining exciton coherence from the photoluminescence spectral line shape in poly(3-hexylthiophene) thin films. Jour. Chem. Phys. 130, 074904 (2009)

29. Ostrowski, D. P. et al. The effect of aggregation on electronic and optical properties of oligothiophene particles. ACS Nano 6, 5507-5513 (2012)

30. Gaufrès, E. et al. Hyperspectral Raman imaging using Bragg filter of graphene and other low-dimensional materials. J. Raman Spectrosc. 49, 1, 174-172 (2018)

31. Almadori, Y. et al. Chromophore ordering by confinement into carbon nanotubes. J. Phys. Chem. C, 118, 19462-19468 (2014)

32. Belhboud, A. et al. Enhancing the infrared response of carbon nanotubes from oligoquaterthiophene interactions. J. Phys. Chem. C, 120, $28802-20807$ (2016)

33. Skowron, S. et al. Chemical Reactions of Molecules Promoted and Simultaneously Imaged by the Electron Beam in Transmission Electron Microscopy. Acc. Chem. Res. 50, 8, 1797$1807(2017)$

34. Jelley, E. E. Spectral absorption and fluorescence in the molecular state. Nature, 138, 1009 $1010(1936)$

35. Spano, F. C. Excitons in conjugated oligomers aggregates, films and crystals. Annu. Rev. Chem, 57, $217-243(2006)$

36. Siciliano, A. Gesuele, R. Pagano, \& G. Guida, M. How Daphnia (Cladocera) assays may be use as bioindicators for health effects? J. Biodivers. Endanger Species S1, 005 (2015) 
37. Celis-Salgado, M. P. Cairns, A. Kim, N. \& Yan, N. D. The FLAMES: a new, softwaterculture and bioassay medium for Cladocera. Verh. Internat. Verein. Mimnol. 30, 2, 265 $271(2008)$

38. Churchman, J. W. Intravenous use of dyes. JAMA 85, 241849 - 1853 (1925)

39. Welsher, K. et al. A route to brightly fluorescent carbon nanotubes for near-infrared imaging in mice. Nature Nanotech. 4, 11, 773 - 780 (2009)

40. Welsher, K. Sherlock, S. P. \& Dai, H. Deep-tissue anatomical imaging of mice using carbon nanotube fluorophores in the second near-infrared window. Proc. Natl. Acad. Sci. 108, 22 , $8943-8948(2011)$

41. Danné, N. et al. Comparative Analysis of Photoluminescence and Up conversion Emission from Individual Carbon Nanotubes for Bioimaging Applications. ACS Photonics 5, 2, 359 $364(2018)$

42. Roxbury, D. et al. Hyperspectral Microscopy of Near-Infrared Fluorescence Enables 17Chirality Carbon Nanotube Imaging. Sci. Rep. 5, 14165 (2015)

43. Shi, L. Sordillo, L. A. Rodriguez-Contreras, A. \& Alfano, R. Transmission in near-infrared windows for deep brain imaging. J. Biophotonics, 9, 1, 38-43 (2016)

44. Antaris, A. L. et al. A small-molecule dye for NIR-II imaging, Nat. Mater. 15, $235-242$ (2016)

45. Fakhri, N. Mackintosh, F.C. Lounis, B. Cognet, L. \& Pasquali, M. Brownian motion of stiff filaments in a crowded environment. Science, 330, 6012, $1804-1807$ (2010)

46. Godin, A. G. Single-nanotube tracking reveals the nanoscale organization of the extracellular space in the live brain. Nat. Nanotechnol. 12, 3, 238 - 243 (2017) 
47. Velayudham, S. et al. Noncovalent Functionalization of Boron Nitride Nanotubes with Poly( p-phenylene-ethynylene)s and Polythiophene. ACS Appl. Mater. Interfaces 2, 1, 104-110 (2010)

48. Ciofani, G. et al. A simple approach to covalent functionalization of boron nitride nanotubes. J. Colloid Interface Sci. 374, 1, 308-314 (2012)

49. Niskanen, J. et al. Boron nitride nanotubes as vehicles for intracellular delivery of fluorescent drugs and probes. Nanomedicine 11, 5, 447-463 (2016) 
Supplementary information file for:

\section{Photostable Fluorescence Imaging Using Dye Nanohybrids}

Charlotte Allard ${ }^{1}$, Rafaella Nascimento ${ }^{2}$ Frédéric Fossard $^{3}$, Léonard Schué ${ }^{2}$, Emmanuel Flahaut $^{4}$, Annick Loiseau ${ }^{3}$, Patrick Desjardins ${ }^{1},{\text { Richard } \text { Martel }^{2 *} \text { and Etienne Gaufrès }}^{3,5 *}$

${ }^{1}$ Regroupement Québécois sur les Matériaux de Pointe (RQMP) and département de génie physique, Polytechnique Montréal, Montréal, Québec H3C 3A7, Canada

${ }^{2}$ Regroupement Québécois sur les Matériaux de Pointe (RQMP) and département de chimie, Université de Montréal, Montréal, Québec H3C 3J7, Canada

${ }^{3}$ Laboratoire d'Etude des Microstructures, ONERA-CNRS, UMR104, Université ParisSaclay, BP 72, 92322 Châtillon Cedex, France

${ }^{4}$ CIRIMAT, Université de Toulouse, CNRS, INPT, UPS, UMR CNRS-UPS-INP N5085, Université Toulouse 3 Paul Sabatier, Bât. CIRIMAT, 118, route de Narbonne, 31062 Toulouse cedex 9, France

${ }^{5}$ Institut d'Optique \& CNRS, LP2N UMR 5298, F-33400 Talence, France

*Correspondence to: etienne.gaufres@u-bordeaux.fr and r.martel@umontreal.ca 


\section{Materials}

1.1 Synthesis of Dyes@BNNTs

1.2 Experiments with Daphniids

2 Methods

2.1 Cleaning the BNNTs.

2.2 Encapsulation of sexithiophene (6T) and DPP molecules in BNNT powder

2.3 Aminopropyltriethoxysilane substrate (APTES) on patterned oxide

2.4 Deposition of BNNTs on APTES patterned substrate.

2.5 Encapsulation of 6T and DPPx into BNNTs deposited on $\mathrm{Si} / \mathrm{SiO}_{2}$ substrate

2.6 Encapsulation of 6T into BNNT on TEM grid (Mo/SiO $/ \mathrm{Cu} / \mathrm{Carbon})$

2.7 Photobleaching experiments of 6T@BNNT and DPP2@BNNT

2.8 Incubation of 6T@BNNT and DPP3@BNNT in Daphnia

2.9 Photobleaching experiments on dye@BNNT assemblies

3 Characterization

3.1 Characterization by AFM

3.2 Characterization by Raman and Photoluminescence.

3.3 Characterization by HR-TEM and TEM-EELS

\section{References}

\subsection{Materials}

1.1 Synthesis of Dyes@BNNTs. The boron nitride nanotubes (BNNT) used in this work were provided by $\mathrm{BNNT}^{\mathrm{TM}}$. Only reagent grade solvents were used. 3-aminopropyltriethoxysilane (APTES) (99\%) and $\alpha$-sexithiophene (6T) were purchased from Sigma-Aldrich and used as 
received. Oligothiophene derivatives such as 3,6-Bis-[2,2']bithiophenyl-5-yl-2,5-di-n-octylpyrrolo[3,4-c]pyrrole-1,4-dione (DPPX) were synthesized according to the procedure (1).

1.2 Experiments with Daphniids. Daphniids used in this work are Daphnia Pulex clones labeled DISP1312 - Disputed Pond in Ontario (coordinates: 42.17N, 83.03W), hence the DISP, and clone \#1312. The culture medium, FLAMES, was prepared similarly to (2). DSPEmPEG,(methoxy-poly(ethyleneglycol)-1,2-distearoyl-sn-glycero-3-phosphoethanolamine-N conjugates) (m.w. 5000) was purchased from Sigma-Aldrich. Adhesive glass slides were purchased from Surgipath Leica biosystems (model APEX Superior). Pre-cleaned white glass micro slides $(25.5 \mathrm{~mm} \times 75.5 \mathrm{~mm} \times 1 \mathrm{~mm})$ were used.

\section{Methods}

\subsection{Cleaning the BNNTs.}

\section{Cleaning of BNNT powder}

The BNNT powder was sonicated in dimethylformamide (DMF) until complete dispersion ( $2 \mathrm{~h})$, and filtrated on a PTFE filter $(0.2 \mu \mathrm{m}$ pore size $)$. The obtained film was annealed at $800^{\circ} \mathrm{C}$ for $2 \mathrm{~h}$ under atmospheric conditions. After the cleaning procedure, the parasite luminescence signal from $\mathrm{BN}$ impurities, $\mathrm{B}_{2} \mathrm{O}_{3}$, and defects became negligible. (see figure $\mathrm{S} 1$ )

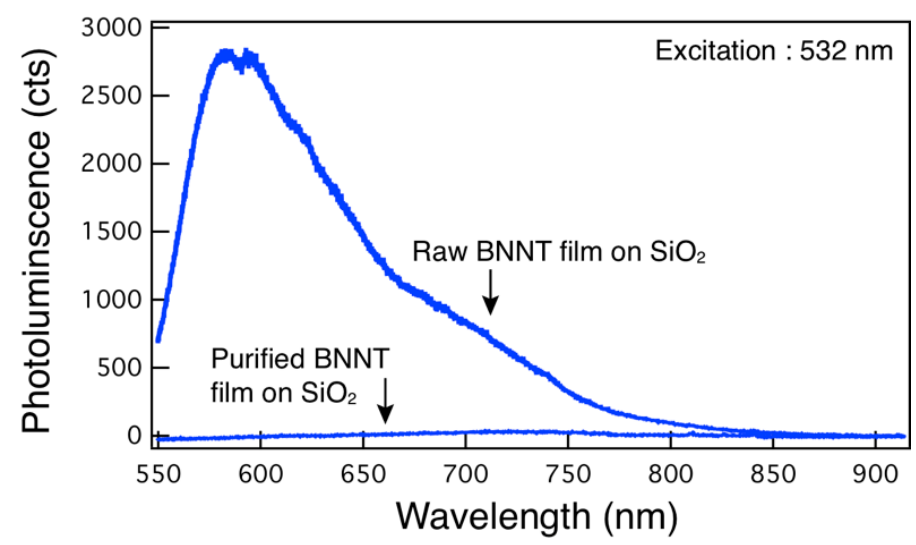


Figure S1: Photoluminescence spectra of BNNT deposited on $\mathrm{Si} / \mathrm{SiO}_{2}$ before and after purification. Each spectrum corresponds to the average contribution from 5 areas on each sample. The wavelength excitation is $532 \mathrm{~nm}$.

\subsection{Encapsulation of sexithiophene (6T) and DPP molecules in BNNT powder.}

6T@BNNT: In a typical experiment, $5 \mathrm{mg}$ of 6T was added to $300 \mathrm{~mL}$ of toluene and dispersed by a mix of stirring and sonication. $20 \mathrm{mg}$ of purified BNNT powder was added to the encapsulation solution and refluxed at $115^{\circ} \mathrm{C}(6 \mathrm{~T})$ for $24 \mathrm{~h}$. The solution was then washed in toluene and DMF at least 10 times to remove the free $6 \mathrm{~T}$, until the filtrate became colorless. Finally, the sample was dispersed in DMF at the appropriate concentration for subsequent characterization.

DPPx@BNNT: $5 \mathrm{mg}$ of DPPX was solubilized in $20 \mathrm{~mL}$ of toluene and $5 \mathrm{mg}$ of purified BNNTs were added to the encapsulation solution. The solution was sonicated for 30 minutes and refluxed at $80^{\circ} \mathrm{C}$ overnight. The washing and dispersion steps are as described above.

\subsection{Aminopropyltriethoxysilane substrates (APTES) on silicon/silicon oxide. The} substrates were cleaned by successive sonication of 10 minutes each in acetone and isopropanol (IPA). The substrates were then immersed in piranha solution for $20 \mathrm{~min}$, rinsed in water, and dried with $\mathrm{N}_{2}$. Finally, the substrates were placed on glass slides suspended above a crystallization dish containing $1 \mathrm{~mL}$ of APTES. The desiccator was vacuum pumped for one minute and the chamber was sealed for an additional thirty seconds. The APTES layer was annealed in air for 45 minutes at about $100{ }^{\circ} \mathrm{C}$ in a conventional oven.

\subsection{Deposition of BNNTs on APTES substrate.}

Raw BNNTs were suspended in $N, N$-dimethylformamide (DMF) and diluted as needed, typically at a concentration of $\sim 0.1 \mathrm{mg} / \mathrm{mL}$. This solution was spin-coated at $3000 \mathrm{rpm}$ on a 
APTES substrate. Atomic Force Microscopy was used to control the quality of the BNNT deposition. See figure S2
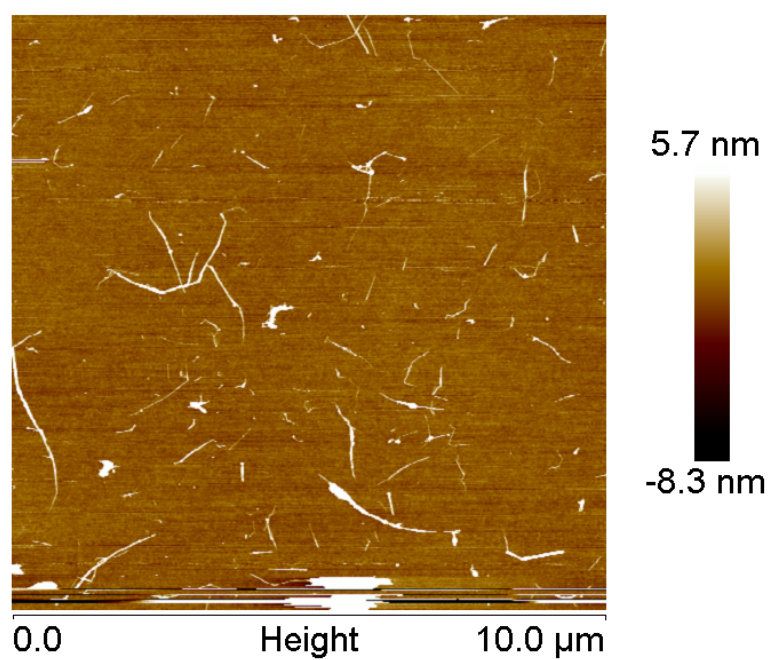

Figure S2: $10 \times 10 \mu \mathrm{m}^{2}$ AFM image of BNNT deposited on $\mathrm{Si} / \mathrm{SiO}_{2}$ substrate after purification.

\subsection{Encapsulation of 6T and DPPx into BNNTs deposited on $\mathrm{Si} / \mathrm{SiO}_{2}$ substrates.}

The sample was treated with concentrated nitric acid for $5 \mathrm{~min}$ and then baked at $800^{\circ} \mathrm{C}$ under vacuum to remove water. The sample was then immediately used for encapsulation. The substrate covered with BNNTs was gently placed into a flask equipped with a condenser and the solution was refluxed overnight at $115{ }^{\circ} \mathrm{C}(6 \mathrm{~T})$ or $80^{\circ} \mathrm{C}$ (DPPX). Following the encapsulation step, the sample was rinsed in fresh toluene, then IPA, and finally dried in $\mathrm{N}_{2}$.

\subsection{Encapsulation of 6T into BNNTs on TEM grid $\left(\mathrm{Mo} / \mathrm{SiO}_{2}\right)$}

$5 \mathrm{mg}$ of raw powder of BNNTs was first mechanically grinded then dispersed into $15 \mathrm{ml}$ of DMF using an ultrasonic bath for one hour. About $20 \mu \mathrm{L}$ of this solution was drop-casted on a Molybdenum grid with holed $\mathrm{SiO}_{2}$ membrane. The deposited BNNTs were then annealed at $350^{\circ} \mathrm{C}$ under atmospheric conditions, followed by another anneal under vacuum at $800^{\circ} \mathrm{C}$ during two hours. The grid was inserted in the $6 \mathrm{~T}$ encapsulation solution at $115^{\circ} \mathrm{C}$ during 6 hours. Following the encapsulation, the grid was rinsed for a few seconds in DMF, cleaned 
using an oxygen plasma $(100 \mathrm{~W}, 10$ minutes $)$ and a piranha treatment (2 minutes) to completely remove the excess of non-encapsulated dyes.

\subsection{Photobleaching experiments of 6T@BNNTs and DPP2@BNNTs}

BNNTs were first deposited on $\mathrm{Si} / \mathrm{SiO} 2$ substrates then encapsulated with $6 \mathrm{~T}$ or DPP2 molecules using the protocol described in section 2.6. Then the integrated intensity of tracked isolated Dyes@BNNT was recorded using the hyperspectral Raman/luminescence imager RIMA $^{\mathrm{TM}}$ from Photon etc. at an excitation wavelength of $532 \mathrm{~nm}$. The time acquisition is 0.2 sec and the laser fluence is $1.2 \mu \mathrm{W} . \mu \mathrm{m}^{-2}$.

\subsection{Incubation of Daphnia Pulex with 6T@BNNTs and DPP3@BNNTs.}

Why Daphnia ? Daphnia is a freshwater crustacean and one of the most studied subjects in Biology, including a fully sequence of its genome, a well-understood life cycle and its ecology. Daphniids are keystone organisms for environmental toxicity studies because they are considered as primary consumer in aquatic food chains (4). In our study, we chose Daphnia because they are filter feeders that conduct small suspended particles into their mouth by the current created by the motion of their leaf-like legs, which makes Daphnia an ideal candidate for our study on BNNTs or dyes@BNNTs suspended in the culture medium of daphniids (FLAMES). Furthermore, in optimal environmental conditions, daphniids reproduce by cyclical parthenogenesis, by generation of diploid eggs that develop directly into larvae at the female brood chamber. The parthenogenesis entails clonal reproduction. The sexual reproduction of daphniids is trigged by environmental stress (e.g. crowding, cooling, changes of photoperiod), which was avoided in our study. Moreover, we used only individuals which did not present any eggs or resting eggs or any larvae in the brood chamber. Furthermore, the maximum incubation time of daphniids with our samples (BNNTs, 
DPPx@BNNTs,6T@BNNTs) was 12 hours, which is not enough to affect the reproduction cycle of 3 days. Daphnia is an ideal system to study the PL from dyes@BNNTs because of its feeding and reproduction systems, which allows us to have identical individuals in the laboratory.

\section{Experiments with dyes@BNNTs in Daphnia.}

\section{A) Preparation of 6T,6T@BNNTs, DPPx@BNNTs and BNNTs in FLAMES:}

For interaction with Daphniids, BNNTs and dye@BNNTs were filtrated and rinsed in FLAMES at least 3 times. $1 \mathrm{mg}$ of each sample was then dispersed in a solution of $200 \mu \mathrm{M}$ mPEG-DSPE in FLAMES, adapted from a previous report (3), and diluted as needed.

\section{B) Incubation of 6T,6T@BNNTs and DPPx@,BNNTs with Daphnia Pulex:}

Adult individuals of Daphnia Pulex (clones DISP1312) were kept at $22^{\circ} \mathrm{C}$ in FLAMES with algae for 4 days before the experiment, allowing a new reproducing cycle. Then, the specimens were transferred to a FLAMES medium algae-free and remained there for 1 day at minimum to clean the digestive system and limit any PL from algae during the images/time lapse acquisition - See Figure S3.
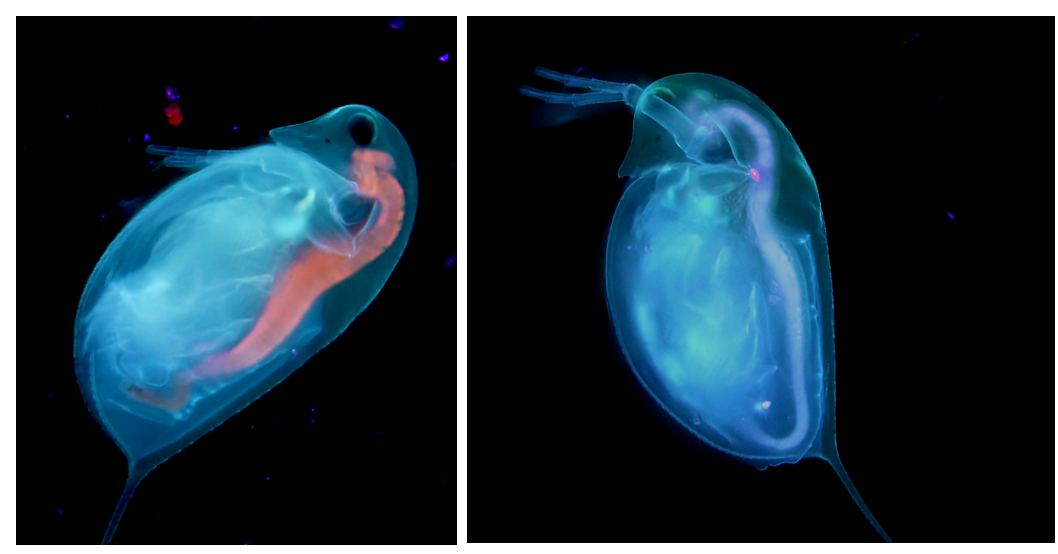

Figure S3. Luminescence image using UBG filter of Daphnia before (left) and after (right) cleaning of digestive tube. 
Daphniids were divided into 3 control groups, and 5 additional groups as described in the Table S1 below. Daphniids were transferred to 8 vials according to Table S1.

Table S1: Description of the groups of Daphniids created for this study by type of sample used for the incubation

\begin{tabular}{|c|c|c|c|}
\hline $\begin{array}{l}\text { Group of } \\
\text { Daphniids }\end{array}$ & $\begin{array}{l}\text { Group of materials dispersed in } \\
\text { algae-free FLAMES, in which } \\
\text { Daphniids were incubated }\end{array}$ & $\begin{array}{l}\text { Concentration } \\
(\mu \mathrm{g} / \mathrm{mL})\end{array}$ & Main observations \\
\hline $\mathrm{A}$ & BNNT dispersed in mPEG-DSPE & $5 ; 25 ; 50 ; 100$ & $\begin{array}{c}\text { Individuals studied at } \\
\text { concentrations } 5 \text { to } 100 \mu \mathrm{g} / \mathrm{mL} \\
\text { were viable }\end{array}$ \\
\hline$* \mathrm{~B}$ & free $6 \mathrm{~T}$ & 5 & $\begin{array}{c}\text { Death of daphniids in a few } \\
\text { minutes }\end{array}$ \\
\hline$* * \mathrm{C}$ & $\begin{array}{c}\text { 6T@BNNTs dispersed in mPEG- } \\
\text { DSPE }\end{array}$ & $5 ; 25 ; 50 ; 100$ & $\begin{array}{c}\text { Individuals studied at } \\
\text { concentrations } 5 \text { to } 50 \mu \mathrm{g} / \mathrm{mL} \\
\text { were viable. Individuals treated } \\
\text { with } 100 \mu \mathrm{g} / \mathrm{mL} \text { had probes } \\
\text { aggregation in the digestive } \\
\text { system, observed by optical } \\
\text { microscope, dying after } 20 \\
\text { hours. }\end{array}$ \\
\hline $\mathrm{D}$ & $\begin{array}{c}\text { DPP2@BNNTs dispersed in } \\
\text { mPEG-DSPE }\end{array}$ & $5 ; 25 ; 50$ & $\begin{array}{c}\text { Individuals studied at } \\
\text { concentrations } 5 \text { to } 50 \mu \mathrm{g} / \mathrm{mL} \\
\text { were viable }\end{array}$ \\
\hline $\mathrm{E}$ & $\begin{array}{c}\text { DPP3@BNNTs dispersed in } \\
\text { mPEG-DSPE }\end{array}$ & $5 ; 25 ; 50$ & $\begin{array}{c}\text { Individuals studied at } \\
\text { concentrations } 5 \text { and } 25 \mu \mathrm{g} / \mathrm{mL} \\
\text { were viable. Individuals treated } \\
\text { with } 50 \mu \mathrm{g} / \mathrm{mL} \text { had some probes } \\
\text { aggregation in the digestive } \\
\text { system, observed by optical } \\
\text { microscope, but when } \\
\text { transferred to algae-free } \\
\text { FLAMES, the daphniids } \\
\text { recovered completely. }\end{array}$ \\
\hline Control 1 & $\begin{array}{l}\text { algae-free FLAMES used to } \\
\text { observe any inherent } \\
\text { photoluminescence of Daphnia in } \\
\text { the conditions of our study }\end{array}$ & - & $\begin{array}{l}\text { Death by starvation after 3-5 } \\
\text { days }\end{array}$ \\
\hline Control 2 & $\begin{array}{c}\text { mPEG-DSPE in algae-free } \\
\text { FLAMES }\end{array}$ & $5 ; 25 ; 50$ & Individuals were viable \\
\hline Control 3 & $\begin{array}{l}\text { algae-free FLAMES to observe the } \\
\text { maximum period during which the } \\
\text { individuals would resist before } \\
\text { death by starvation }\end{array}$ & - & $\begin{array}{l}\text { Death by starvation after 3-5 } \\
\text { days }\end{array}$ \\
\hline
\end{tabular}

* The concentration of $6 \mathrm{~T}$ was limited to $5 \mathrm{ug} / \mathrm{mL}$ due the low capacity of dispersion of the dye in the FLAMES. 
** After the excess of aggregation of the probes 6T@BNNTs-mPEG-DSPE, we decided to limit the maximum concentration to $50 \mathrm{ug} / \mathrm{mL}$

\section{C) PL measurements of 6T,6T@BNNTs, DPPX@,BNNTs and BNNTs with Daphnia Pulex:}

To perform the PL measurements, each Daphnia was transferred with the incubation medium to a glass slide. The excess of liquid was dried out in order to keep the minimum necessary to maintain Daphnia alive. Following, movies of the PL from dyes@BNNTs in Daphnia were acquired using a UBG filter. Longer PL measurements (from $\mathrm{t}=0$ to $\mathrm{t}=12$ hours) had a duplicate slide on which the liquid was dried out and daphniids were kept in air.

The NIR PL image in Figure 4D was performed using a Daphnia individual incubated with

DPP3@BNNTs $(5 \mu \mathrm{g} / \mathrm{ml})$ and recorded using the Hyperspectral Raman/PL Imager - RIMA ${ }^{\mathrm{TM}}$ from Photon etc (laser power of $500 \mathrm{~mW}, 10 \mathrm{X}$ objective, acquisition time $2 \mathrm{sec}$ ), equiped with a $900 \mathrm{~nm}$ long-pass filter.

D) Evaluation of potential toxicity and reverse cycle of incubation of Daphnia with

\section{T@BNNTs, DPP3@BNNTs and BNNTs:}

In order to evaluate the potential toxicity of our samples (6T,6T@BNNTs, DPP2@BNNTs, DPP3@BNNTs and BNNTs), we kept 3 individuals of each group described in Table S1. Daphniids were incubated for a period of 36 hours and there was no observed death of individuals, except from group B (incubated with free $6 \mathrm{~T}$ at $5 \mu \mathrm{g} / \mathrm{mL}$ ) who died after a few minutes of incubation, and group C (with 6T@BNNT at $100 \mu \mathrm{g} / \mathrm{mL}$ ) who died after 20 hours of incubation due to aggregation of the probe in the digestive system. Next, to evaluate the capacity of daphniids to get rid of the 6T@BNNTs, DPP2@BNNTs and BNNTs after the 36h incubation experiment, we took living individuals of groups A, C, D, E and of Control 2 and transferred them to clean algae-free FLAMES (for 2 hours) followed by FLAMES/algae 
medium. All the individuals survived after the end of the experiment. Further, they were monitored for more than 30 days after a full cycle of reproduction.

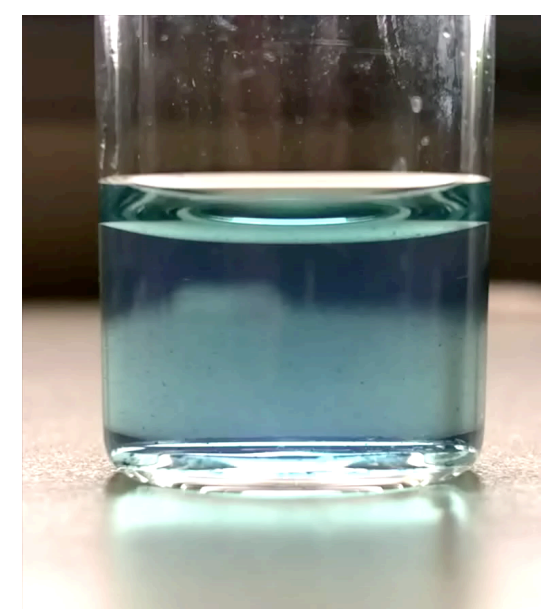

Figure S4. Video of a living Daphnia after an incubation of 12 h in a DPP2@BNNT solution.

\subsection{Photobleaching experiments on dye@BNNT assemblies}

The stability of the dye@BNNT was assessed in solution, and compared to the dye alone, after an overnight exposition to a conventional lamp - see Figure S5
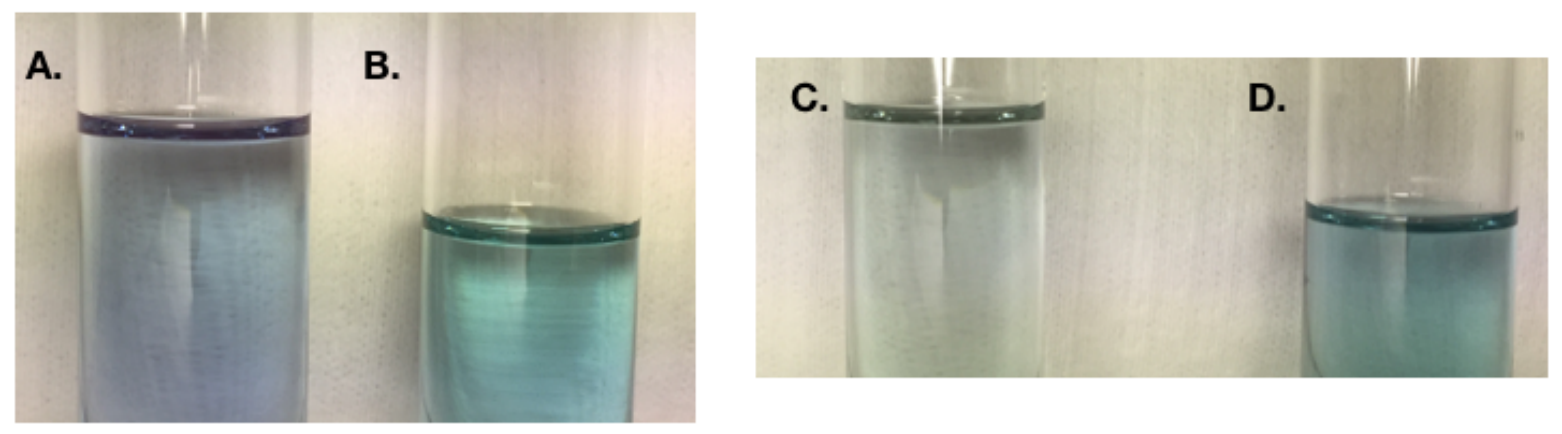

Figure S5. A.) DPP2 in DMF B.) DPP2@BNNTs in DMF C.) DPP2 in DMF after overnight light exposition D.) DPP2@BNNTs in DMF after overnight light exposition. Slight changes in lighting can account for the color difference of the DPP2@BNNTs. 


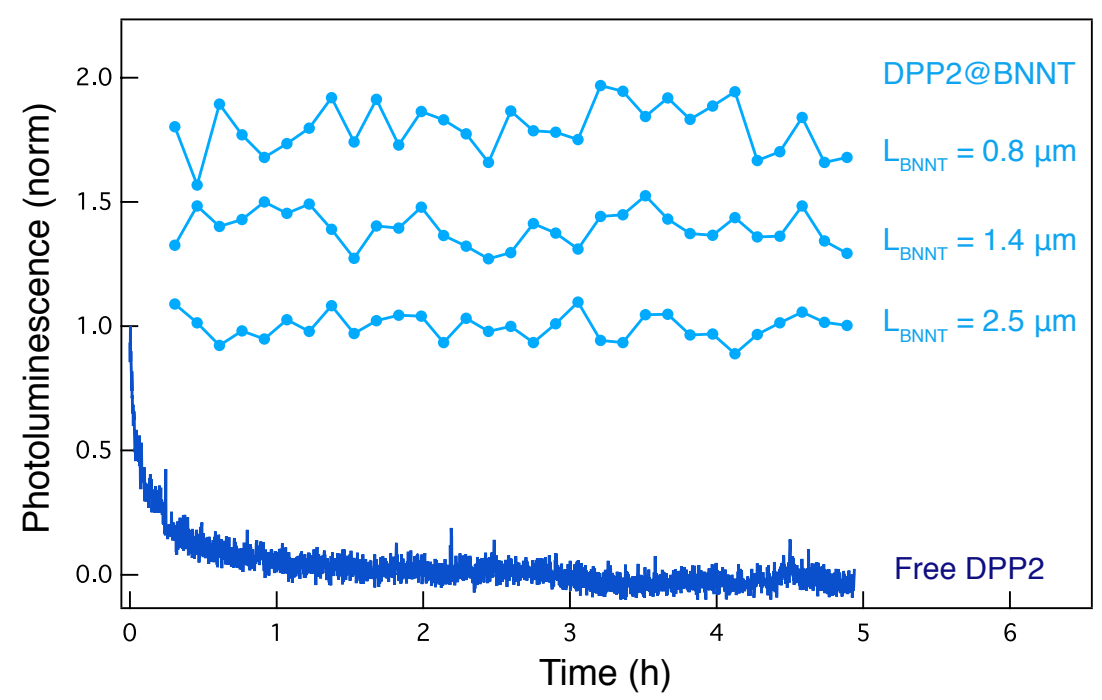

Figure S6. Photoluminescence of isolated DPP2@BNNT deposited on Si/SiO2 substrate as a function of time, and compared to free DPP2 molecules deposited on the same substrate. The excitation wavelength $\lambda_{\text {ex }}=532 \mathrm{~nm}$ and the laser fluence is $1.2 \mu \mathrm{W} \cdot \mu \mathrm{m}^{-2}$.

\section{Characterization}

3.1 Characterization by AFM. The AFM images were produced using a Dimension 3100 scanning probe microscope equipped with a Nanoscope IV controller and a quadrex extender module. Height images were acquired using intermittent-contact mode using silicon probes from APP-Nano, of nominal spring constants ranging from $13 \mathrm{Nm}^{-1}-77 \mathrm{Nm}^{-1}$, resonance frequency ranging from $200 \mathrm{kHz}-400 \mathrm{kHz}$, and tip radius curvature $<10 \mathrm{~nm}$.

3.2 Characterization by Raman and photoluminescence. The Raman/PL measurements were taken from different set-up depending on the experiment. The equipment of the different set-ups is summarized in Table S2 and are described as follows:

- The Luminescence images and photobleaching experiments presented in Figure 3 were performed at $532 \mathrm{~nm}$ using the Raman/PL mapping system RIMA ${ }^{\mathrm{TM}}$ from Photon etc. In the Luminescence mode imaging the signal on the map is the integrated intensity of the 
photoluminescence using a 100X objective and a laser power ranging from $0.5 \mu \mathrm{W} . \mu \mathrm{m}^{-2}$ to 80 $\mu \mathrm{W} \cdot \mu \mathrm{m}^{-2}$

- The PL spectra in Figure 3D were acquired on a Raman/PL Renishaw Spectrometer

- The optical imaging of Daphnia Pulex presented in the panel \#1 in Figure 4 was done using a confocal microscope. A z-stack of 20 images was acquired to probe the volume of the daphnia and then the Auto-blend function in Phostoshop was applied to reconstruct the image.

- The fluorescence images in Figure 4 were acquired with an Olympus confocal microscope equipped with a U-RFL-T mercury light source, a BX-UCB controller and a DP71 digital camera. The images were acquired with a $10 \mathrm{X}$ objective and UBG, TRITC and TXRED filters. The acquiring conditions were: Exposure time: 1/20 sec; ISO sensitivity: ISO 200;

Table S2. Raman/PL measurements setup used for the experiments

\begin{tabular}{|c|c|c|c|c|}
\hline Set up & Excitation & Objective & $\begin{array}{c}\text { Dispersive } \\
\text { element }\end{array}$ & Detection \\
\hline $\begin{array}{c}\text { Custom optical } \\
\text { bench }\end{array}$ & $\begin{array}{c}\text { Verdi } 532 \mathrm{~nm} \\
\text { HeNe } 633 \mathrm{~nm}\end{array}$ & $\begin{array}{c}\text { Olympus 50X } \\
\text { long working } \\
\text { distance NA } \\
0.55\end{array}$ & $\begin{array}{c}\text { Grating, 600 } \\
\text { and } 1800 \mathrm{tt} / \mathrm{mm}\end{array}$ & $\begin{array}{c}\text { CCD JY } \\
\text { nitrogen cooled }\end{array}$ \\
\hline RIMA & Opus 532 nm & Olympus 100X & $\begin{array}{c}\text { no } \\
\text { (broadband } \\
\text { mode) optical } \\
\text { filters }\end{array}$ & $\begin{array}{c}\text { CCD Pixis } \\
\text { (Princeton Inst.) } \\
\text { Peltier }\end{array}$ \\
\hline $\begin{array}{c}\text { Renishaw InVia } \\
\text { Reflex }\end{array}$ & Cobolt 473nm & Olympus 50X & $\begin{array}{c}\text { Grating, 1200 } \\
\text { and 2400 tt/mm }\end{array}$ & $\begin{array}{c}\text { CCD Renishaw } \\
\text { RemCam, } \\
\text { Peltier }\end{array}$ \\
\hline $\begin{array}{c}\text { Fluorescence } \\
\text { microscope }\end{array}$ & $\begin{array}{c}\text { U-RFL-T } \\
\text { mercury light } \\
\text { source }\end{array}$ & $10 \mathrm{X}$ & $\begin{array}{c}\text { nBG, TRITC } \\
\text { and TXRED } \\
\text { filters }\end{array}$ \\
\hline
\end{tabular}

3.3 Characterization by HR-TEM and TEM-EELS TEM and high-angle annular dark-field (HAADF) STEM images in Figure 1 and S2a-b were recorded with a Libra 200 MC Zeiss operating at $200 \mathrm{kV}$ and at $80 \mathrm{kV}$. The microscope is equipped with a monochromatic 
Schottky FEG source delivering an energy resolution down to $150 \mathrm{meV}$ and a Gatan Ultrascan camera. Point resolution is $2.3 \AA$ and information limit is $1.2 \AA$ at $200 \mathrm{kV}$. The TEM can operate in scanning mode (STEM) and is equipped with Bright Field (BF) and HAADF detectors. The instrument is also equipped with an in-column energy filter making possible energy filtered TEM (EFTEM), EELS measurements in Scanning TEM mode and acquisition of spectrum imaging datacubes (or hyperspectral EELS). The STEM-HAADF probe size is about $2.5 \mathrm{~nm}$ (Figure S7).
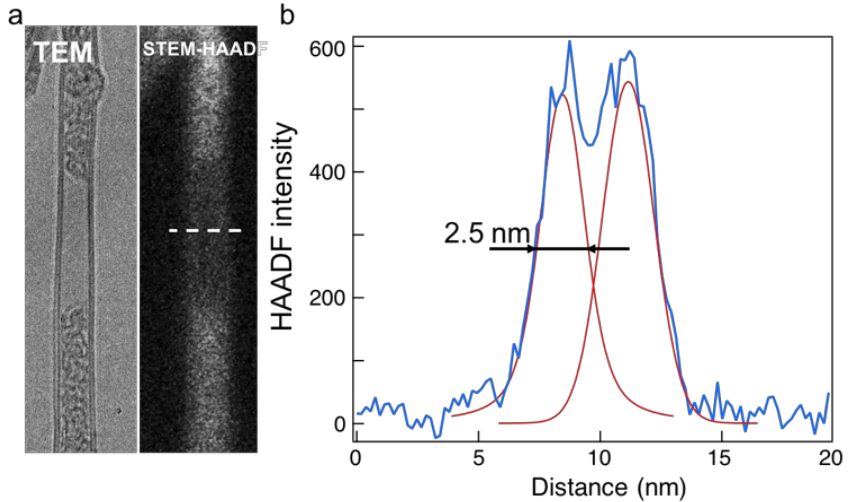

Figure S7: a TEM and STEM-HAADF (left and right respectively) images at $80 \mathrm{kV}$ of a double walled boron nitride partially filled with $6 \mathrm{~T}$ molecules. b HAADF profile of the empty part of the BNNT along the cross section dashed line indicated in (a). The deconvolution of the wall gives a special resolution of about $2.5 \mathrm{~nm}$ of the STEM probe at 80 $\mathrm{kV}$

The High Resolution images presented in Figure $3 \mathrm{~F}$ and $\mathrm{S} 8$ were performed at $80 \mathrm{kV}$ on a JEOL ARM microscope, equipped with an aberration corrector. 


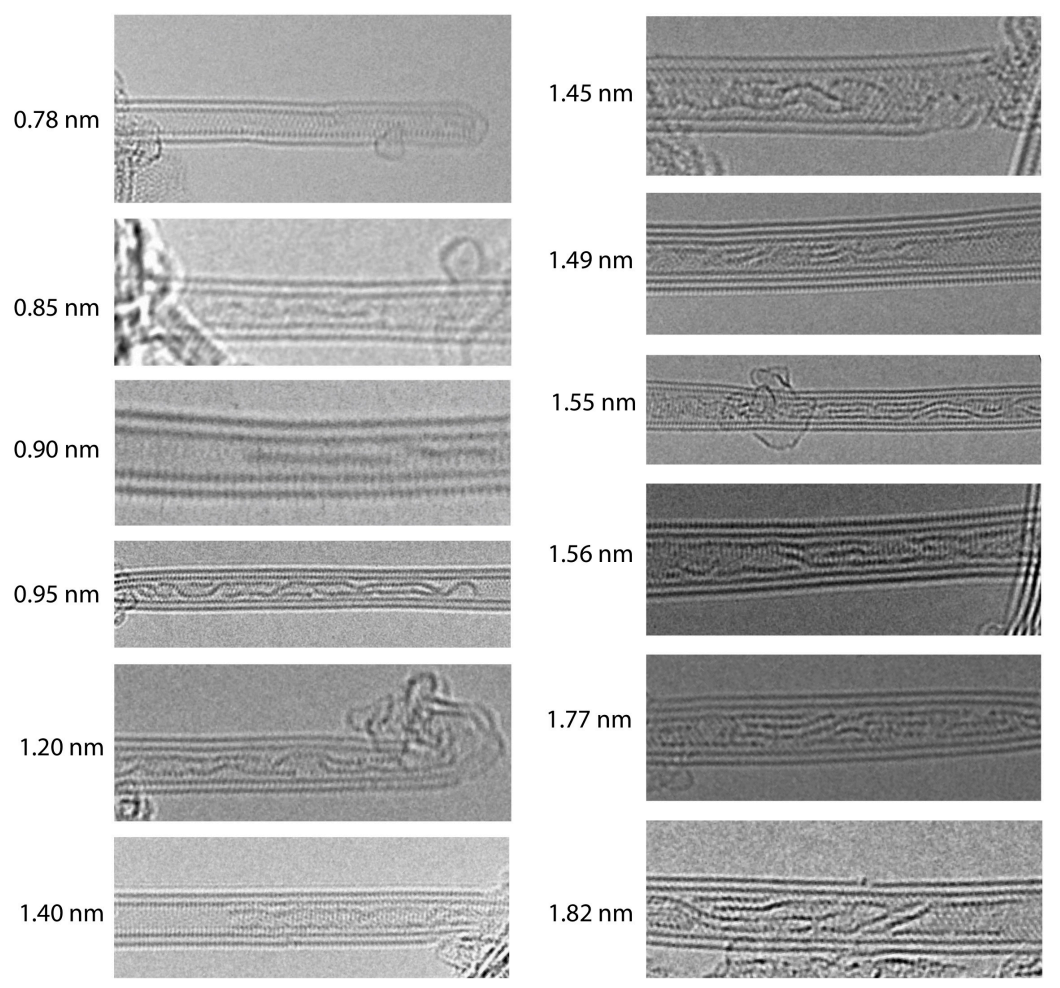

Figure S8: High Resolution image recorded at $80 \mathrm{kV}$ of $6 \mathrm{~T} @ \mathrm{BNNT}$ for various inner diameters, highlighting the progressive ordering of the molecules as a function of the 1D confinement. Most of the BNNT observed are filled or partially filled with molecules. Only the observed BNNT having an inner diameter bellow $0.8 \mathrm{~nm}$ are non-encapsulated, that represents probably the diameter limit for encapsulating 6T molecules in BNNT.

\section{References}

(1) A. B. Tamayo, M. Tantiwiwat, B. Walker, T.-Q. Nguyen, J. Phys. Chem. C, 112, 15543 (2008)

(2) M. P. Celis-Salgado, A. Cairns, N. Kim, \& N.D. Yan, The FLAMES medium: a new, softwater culture and bioassay medium for Cladocera. Internationale Vereinigung für theoretische und angewandte Limnologie: Verhandlungen, 30, 265-271 (2008).

(3) V. H. Lee, D. Zhang, Y. K. Yap, Functionalization, dispersion and cutting of boron nitride in water, J. Phys. Chem. C, 116, 1798-1804 (2012)

(4) A. Siciliano, R. Gesuele, G. Pagano, M. Guida, How Daphnia (cladocera) assays may be used as bioindicators of health effects? J. Biodivers Endanger Species, 1, 5 (2015) 
